\title{
High levels of apoptosis are induced in human glioma cell lines by co-administration of peptide nucleic acids targeting miR-221 and miR-222
}

\author{
ELEONORA BROGNARA $^{1}$, ENRICA FABBRI ${ }^{1}$, GIULIA MONTAGNER ${ }^{1}$, JESSICA GASPARELLO $^{1}$, \\ ALEX MANICARDI ${ }^{2}$, ROBERTO CORRADINI ${ }^{2}$, NICOLETTA BIANCHI ${ }^{1}$, ALESSIA FINOTTI ${ }^{1}$, \\ GIULIA BREVEGLIERI ${ }^{1}$, MONICA BORGATTI ${ }^{1}$, ILARIA LAMPRONTI ${ }^{1}$, ROBERTA MILANI ${ }^{1}$, \\ MARIA CRISTINA DECHECCHI ${ }^{3}$, GIULIO CABRINI ${ }^{3}$ and ROBERTO GAMBARI ${ }^{1}$ \\ ${ }^{1}$ Department of Life Sciences and Biotechnology, University of Ferrara, Ferrara; ${ }^{2}$ Department of Chemistry, \\ University of Parma, Parma; ${ }^{3}$ Laboratory of Molecular Pathology, University-Hospital of Verona, Verona, Italy
}

Received August 7, 2015; Accepted September 4, 2015

DOI: 10.3892/ijo.2015.3308

\begin{abstract}
The biological activity of a combined treatment of U251, U373 and T98G glioma cell lines with two anti-miR PNAs, directed against miR-221 and miR-222 and conjugated with an ocataarginine tail (R8-PNA-a221 and R8-PNA-a222) for efficient cellular delivery, was determined. Apoptosis was analyzed, and the effect of the combined treatment of glioma cells with either or both PNAs on the reversion of drug-resistance phenotype was assessed in the temozolomideresistant T98G glioma cell line. Selectivity of PNA/miRNA interactions was studied by surface plasmon resonance (SPR)based Biacore analysis. Specificity of the PNA effects at the cellular level was analyzed by RT-qPCR. These experiments support the concept that the effects of R8-PNA-a221 and R8-PNA-a222 are specific. The studies on apoptosis confirmed that the R8-PNA-a221 induces apoptosis and demonstrated the pro-apoptotic effects of R8-PNA-a222. Remarkably, increased pro-apoptotic effects were obtained with the co-administration of both anti-miR-221 and anti-miR-222 PNAs. In addition, co-administration of R8-PNA-a221 and R8-PNA-a222
\end{abstract}

Correspondence to: Professor Roberto Gambari, Department of Life Sciences and Biotechnology, University of Ferrara, Via Fossato di Mortara n.74, I-44121 Ferrara, Italy

E-mail: gam@unife.it

Abbreviations: PNA, peptide nucleic acid; Fl, fluorescein; RT-qPCR, reverse transcription quantitative polymerase-chain reaction; SDS, sodium dodecylsulphate; SDS-PAGE, SDS-polyacrylamide-gel electrophoresis; TMZ, temozolomide; PUMA, p53-upregulated modulator of apoptosis; PTEN, phosphatase and tensin homolog; TIMP3, metalloproteinase inhibitor 3; ICAM-1, intercellular adhesion molecule 1; SPR, surface plasmon resonance; HBS, HEPES-buffered saline

Key words: peptide nucleic acids, glioma, microRNAs, miR-221, miR-222, miRNA targeting, delivery induced apoptosis of TMZ-treated T98G cells at a level higher than that obtained following singular administration of R8-PNA-a221 or R8-PNA-a222.

\section{Introduction}

Peptide nucleic acids (PNAs) are DNA analogues in which the sugar-phosphate backbone has been replaced by N-(2aminoethyl)glycine units (1-5). These very interesting molecules were described for the first time by Nielsen et al (1) and, despite a radical structural change with respect to DNA and RNA, they are capable of sequence-specific and efficient hybridization with complementary DNA and RNA, forming Watson-Crick double helices (1). In addition, they are able to generate triple helix formation with double stranded DNA and perform strand invasion (2-4). Accordingly, they have been used as very efficient tools for pharmacologic alteration of gene expression, both in vitro and in vivo (1-5). PNAs and PNA-based analogues were proposed as antisense molecules targeting mRNAs, triple-helix forming molecules targeting eukaryotic gene promoters, artificial promoters, decoy molecules targeting transcription factors (4-10). Recently, PNAs have been shown to be able to alter biological functions of microRNAs, both in vitro and in vivo (11-18). Cheng et al, for instance, demonstrated that attachment of a peptide-(anti-miR) PNA construct is able to target the tumor microenvironment and to transport the anti-miR PNA across plasma membranes under acidic conditions such as those found in solid tumors. This treatment led to an efficient inhibition of the target oncomiR in a tumor mouse model (18).

MicroRNAs (miRNAs or miRs) are short non-coding RNA molecules, which act as gene regulators by repressing translation or by inducing the cleavage of target RNA transcripts (19). Emerging evidence suggests that the altered expression of miRNA may be involved in the pathogenesis of cancer (20-25). Therefore, in the case of oncomiRNAs and metastamiRNAs, approaches based on the targeting of microRNAs can be designed to inhibit tumor cell growth and metastasis, and to counteract the resistance of tumor cells 
to anticancer drugs. Chan et al demonstrated that sequencespecific functional inhibition of oncomiR-138 in malignant gliomas prevents tumor sphere formation in vitro and impedes tumorigenesis in vivo (26). In addition, Wagenaar et al were able to deregulate the transcriptional network in hepatocellular carcinomas by targeting miR-21 with sequence-specific antagomiRs, thus inducing a significant de-repression of direct targets of miR-21 which led to loss of viability in the majority of hepatocarcinoma cell lines tested (27). In another example Ma et al reported that therapeutic silencing of the oncogenic miR-10b inhibits metastasis in a mouse model of mammary tumor (28). In this context, the use of PNAs directed against oncomiRNAs might be of great interest (20).

Among the possible microRNA targets involved in cancer, the cluster miR-221/222 plays a very important role (29-34). Lee et al found that high levels of miR-221/222 cause increased cell invasion and poor prognosis in glioblastomas (33). Accordingly, co-suppression of miR-221/222 is associated with inhibition of cell growth, increased expression of the miR-221/222 targets, in particular of the p53-upregulated modulator of apoptosis (PUMA), and activation of apoptosis (35). Enforced expression of miR-221/222 induces cell survival whereas knockdown of miR-221/222 stimulates cellular apoptosis. Interestingly, the miR-221/222 cluster is also involved in sensitization of glioma cells to anticancer drugs (34). Accordingly, downregulation of miR-221/222 sensitizes glioma cells to temozolomide, regulating apoptosis (34).

We have recently designed and studied a peptide nucleic acid targeting miR-221 (R8-PNA-a221) (36), bearing an oligoarginine peptide (R8) to facilitate uptake by glioma cells $(11,12)$. The effects of the R8-PNA-a221 were analyzed in U251, U373 and T98G glioma cells and found to strongly inhibit miR-221. In addition, the effects of R8-PNA-a221 on $\mathrm{p} 27^{\mathrm{Kip} 1}$ (a target of miR-221) were analyzed in U251 and T98G cells by RT-qPCR and by western blotting. We found an increase of $\mathrm{p} 27^{\mathrm{Kip} 1} \mathrm{mRNA}$ and of $\mathrm{p} 27^{\mathrm{Kip} 1}$ protein in cells treated with R8-PNA-a221 (36).

The present study was aimed at determining the biological activity of a combined treatment of glioma cell lines with two PNAs, directed against miR-221 and miR-222 and conjugated to octaarginine for cellular delivery. Apoptosis was analyzed to determine whether co-administration of the two PNAs is important to obtain the highest effects. The effects of the combined treatment of glioma cells on the reversion of drug-resistance phenotype were assessed in the temozolomideresistant T98G glioma cell line.

\section{Materials and methods}

Synthesis and characterization of PNAs. The synthesis and characterization of anti-miR-221 PNAs have been previously reported (36), the new anti-miR-222 PNAs synthesis was performed using standard Fmoc-based automate peptide synthesizer (Syro II, MultiSynTech GmbH, Witten, Germany), using a ChemMatrix-RinkAmide resin loaded with FmocGly-OH $(0.2 \mathrm{mmol} / \mathrm{g})$ as first monomer and using commercially available monomers (Link Technologies, Bellshill, UK) with HBTU/DIPEA coupling. After purification the PNAs were characterized by UPLC-MS on a Water Acquity System equipped with Acquity UPLC BEH C18 (1.7 $\mu \mathrm{m}, 2.1 \times 50 \mathrm{~mm})$.
Gradient: $100 \%$ A for $0.9 \mathrm{~min}$, then from 0 to $50 \% \mathrm{~B}$ in $5.7 \mathrm{~min}$ at $0.25 \mathrm{ml} / \mathrm{min}$ flow $(\mathrm{A}$, water $+0.2 \%$ formic acid; $\mathrm{B}$, acetonitrile $+0.2 \%$ formic acid).

R8-PNA-a222: $\mathrm{R}_{\mathrm{t}}=2.70 \mathrm{~min}$; calculated mw, 6,226.3 g/ $\mathrm{mol}$; m/z found: $1,246.5[\mathrm{M}+5 \mathrm{H}]^{5+}, 1,039.0[\mathrm{M}+6 \mathrm{H}]^{6+}, 890.4$ $[\mathrm{M}+7 \mathrm{H}]^{7+}, 779.5[\mathrm{M}+8 \mathrm{H}]^{8+}, 693.0[\mathrm{M}+9 \mathrm{H}]^{9+}, 623.8[\mathrm{M}+10 \mathrm{H}]^{10+}$, $567.2[\mathrm{M}+11 \mathrm{H}]^{11+}$.

R8-PNA-a222-MUT: $\mathrm{R}_{\mathrm{t}}=2.70 \mathrm{~min}$; calculated mw, $6,226.34 \mathrm{~g} / \mathrm{mol} ; \mathrm{m} / \mathrm{z}$ found: $1,246.2[\mathrm{M}+5 \mathrm{H}]^{5+}, 1,038.7[\mathrm{M}+6 \mathrm{H}]^{6+}$, $890.3[\mathrm{M}+7 \mathrm{H}]^{7+}, 779.1[\mathrm{M}+8 \mathrm{H}]^{8+}, 692.8[\mathrm{M}+9 \mathrm{H}]^{9+}$.

Bioinformatic tools for molecular interaction studies. Predicted interactions between microRNAs and target mRNA sequences were determined using the following tools: a) the ViennaRNA Web Services (Institute of Theoretical Chemistry, Vienna University), webRNAfold and WebServer (http://rna.tbi.univie.ac.at/), which predict minimum free energy secondary structures and base pair probabilities from single stranded RNA sequences; b) the UCSC (University of California Santa Cruz) Genome Browser (https://genome.ucsc. edu/) Gene Sorter, which shows expression, homology and other information on genes, and it was used for the 3'-UTR base sequence; c) the microRNA database miRBase (University of Manchester, http://www.mirbase.org/), for published miRNA sequences and annotation.

Biospecific interaction analysis (BIA) with the Biacore X100. All procedures were performed at $25^{\circ} \mathrm{C}$ and at a $5-\mu 1 / \mathrm{min}$ flow rate, by using HBS-EP (0.01 M HEPES pH 7.4, 0.15 M NaCl, $3 \mathrm{mM}$ EDTA, $0.005 \%$ v/v Surfactant P20) (GE Healthcare) as running buffer. CM5 sensor chips (GE Healthcare) containing PNAs were obtained by the amine coupling chemistry, exploiting the terminal amine group of the PNA. To this aim, the Amine Coupling kit (GE Healthcare) was used: briefly, carboxyl groups on the sensor chip surface were first activated with a 1:1 mixture of 0.4 M 1-ethyl-3-(3-dimethylaminopropyl)-carbodiimide (EDC) and 0.1 M N-hydroxysuccinimide (NHS) to give reactive succinimide esters; the R8-PNA-a221, R8-PNA-a222, R8-PNA-a221-MUT and R8-PNA-a222-MUT were then passed over the surface at the concentration of $50 \mu \mathrm{g} / \mathrm{ml}$ for $10 \mathrm{~min}$, in order to form a covalent bond; finally, $1 \mathrm{M}$ ethanolamine- $\mathrm{HCl}$ at $\mathrm{pH} 8.5$ was injected to quench the excess of reactive groups. Hybridization to pre-miR-221 and pre-miR-222 were performed in $10 \%$ HBS buffer, followed by washing with HBS and a 1-min pulse of $50 \mathrm{mM} \mathrm{NaOH}$ for regeneration of flow cells. Biacore X100 Control Software and Biacore X100 Evaluation Software, version 2.0.1 (GE Healthcare) were used for operation and data analysis, respectively $(37,38)$.

Glioma cell lines and culture conditions. U251, U373 and T98G cells (39-41) were cultured in humidified atmosphere of 5\% $\mathrm{CO}_{2}$ /air in RPMI-1640 medium (Life Technologies, Monza, Italy) supplemented with $10 \%$ fetal bovine serum (FBS, Celbio, Milan, Italy), $100 \mathrm{U} / \mathrm{ml}$ penicillin and $100 \mathrm{mg} / \mathrm{ml}$ streptomycin. To verify the effect on proliferation, cell growth was monitored by determining the cell number/ml using a Z2 Coulter Counter (Coulter Electronics, Hialeah, FL, USA).

RNA extraction. Cultured cells were tripsinized and collected by centrifugation at $1,500 \mathrm{rpm}$ for $10 \mathrm{~min}$ at $4^{\circ} \mathrm{C}$, washed 
with PBS, lysed with Tri-reagent (Sigma-Aldrich, St. Louis, MO, USA), according to the manufacturer's instructions. The isolated RNA was washed once with cold $75 \%$ ethanol, dried and dissolved in nuclease free pure water before use.

Quantitative analyses of miRNAs. For miRNA quantification using real-time RT-qPCR reagents, the primers and probes (hsa-miR-221, TM:000524, hsa-miR-222, TM:000525) were obtained from Applied Biosystems. Reverse transcriptase (RT) reactions were performed using the TaqMan MicroRNA Reverse Transcription kit (Applied Biosystems, Foster City, CA, USA); real-time PCR was performed according to the manufacturer's protocols (36). Twenty nanograms per sample were used for the assays. All RT reactions, including no-template controls and RT-minus controls, were performed in duplicate using the CFX96 Touch Real-Time PCR Detection System (Bio-Rad, Hercules, CA, USA). The relative expression was calculated using the comparative cycle threshold method and U6 snRNA (TM:001973) and hsa-let-7c (TM:000379) were used as references to normalize all RNA samples, since they remain constant in the assayed samples by miR-profiling and quantitative RT-PCR analysis, as previously reported (36).

Analysis of apoptosis. Annexin V and Dead Cell assay on T98G cells, untreated and treated with temozolomide (Sigma-Aldrich) (36) and different concentrations of PNAs, were performed with Muse (Millipore Corp., Billerica, MA, USA) method, according to the instructions supplied by the manufacturer. This procedure utilizes Annexin V to detect PS (phosphatidylserine) on the external membrane of apoptotic cells. A dead cell marker is also used as an indicator of cell membrane structural integrity. It is excluded from live, healthy cells, as well as early apoptotic cells. Four populations of cells can be distinguished in this assay. Cells were washed with sterile 1X PBS, trypsinized, suspended and diluted (1:2) with the one step addition of the Muse Annexin V \& Dead Cell reagent. After incubation of $20 \mathrm{~min}$ at room temperature in the dark, samples were analyzed. Data from prepared samples are acquired and recorded utilizing the Annexin V and Dead Cell Software Module (Millipore) (36).

Statistical analyses. Results are expressed as mean \pm standard deviation (SD). Comparisons between groups were made by using paired Student's t test. Statistical significance was defined with $\mathrm{p}<0.05$ (significant) and $\mathrm{p}<0.01$ (highly significant).

\section{Results}

Design of the PNA molecules targeting $m i R-221$ and miR-222. We first demonstrated that the human glioma cell lines U251, U373 and T98G express at high levels both miR-221 and miR-222 (Fig. 1). The structure of pre-miR-221 and pre-miR-222, together with their possible site of interaction within the 3'-UTR sequences of PUMA, one of the major mRNA targets of miR-221 and miR-222, is depicted in Fig. 2. The sequences of the PNAs utilized in the present study are reported in Table I. In particular the design and synthesis of the a221 PNAs were already reported (36), while the a222 PNAs were designed to be complementary to the seed region of the mature miR-222 (full complementarity to nt 1-18). Full-

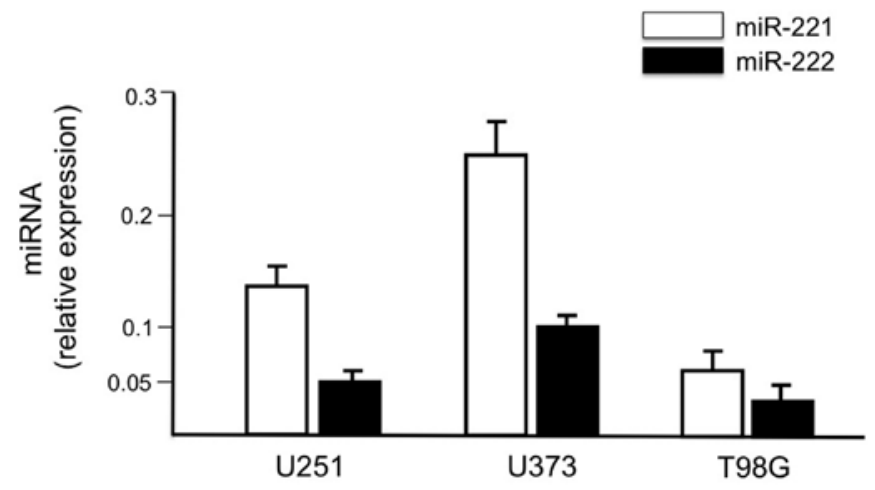

Figure 1. Expression of miR-221 and miR-222 in the human glioma cell lines used in the present study. RNA was isolated from cells cultured for 4 days and RT-qPCR performed. Results are presented as relative values (average $\pm \mathrm{SD}$ in three independent experiments).

matched PNAs are expected to bind to both pre-miR-221 and pre-miR-222 as well as to mature miR-221 and miR-222. An octaarginine peptide ( $\mathrm{R} 8$ ) has been conjugated to the PNAs, since it has been reported that this peptide displays optimal uptake, while shorter oligoarginine sequences (R2, R4, R6) give poorer results $(11,12,36,42,43)$. In order to verify the selectivity of the observed effects, PNAs containing four mismatches were also synthesized, and their design was carried out in order to avoid possible complex formation of the off target sequences.

Validation of the PNA molecules targeting miR-221 and miR-222: a Biacore study. The ability of the R8-PNA-a221 and R8-PNA-a222 to specifically interact with pre-miR-221 and pre-miR-222 has been validated by SPR-based analysis using the Biacore X100 biosensor. Fig. 3A shows representative data obtained by injecting $50 \mathrm{ng}$ of pre-miR-221 on sensor chips to which R8-PNA-a221, R8-PNA-a222, R8-PNA-a221-MUT and the R8-PNA-a222-MUT have been immobilized. The hybridization between pre-miR-221 and R8-PNA-a221 occurs within seconds (and the dissociation is very slow, leading to persistence of high RUres values). On the contrary, pre-miR-221 binds weakly to immobilized R8-PNA-a222 sensor chip. Importantly, the binding of pre-miR-221 to sensor chips on which R8-PNA-a221-MUT and R8-PNA-a222-MUT were immobilized is very low. This experiment suggests that the binding of pre-miR-221 to the R8-PNA-a221 is selective. Similar conclusions can be drawn from the experiment shown in Fig. 3B, which demonstrates that pre-miR-222 binds to R8-PNA-a222 immobilized on the sensor chip much more efficiently than to R8-PNA-a221. However, a certain degree of cross-binging was observed for pre-miR-221 on R8-PNA222 chip (higher than that of R8-PNA-a221-MUT), and for pre-miR-222 on R8-PNA-221 sensor chips. As expected, no binding of pre-miR-210 was found to either R8-PNA-a221 or R8-PNA-a222 (Fig. 3C). Table II summarizes the results of RUfin and RUres obtained when pre-miR-221 and pre-miR-222 were injected in sensor chips to which normal and mutated anti-miR-221 and anti-miR-222 PNAs were immobilized. 


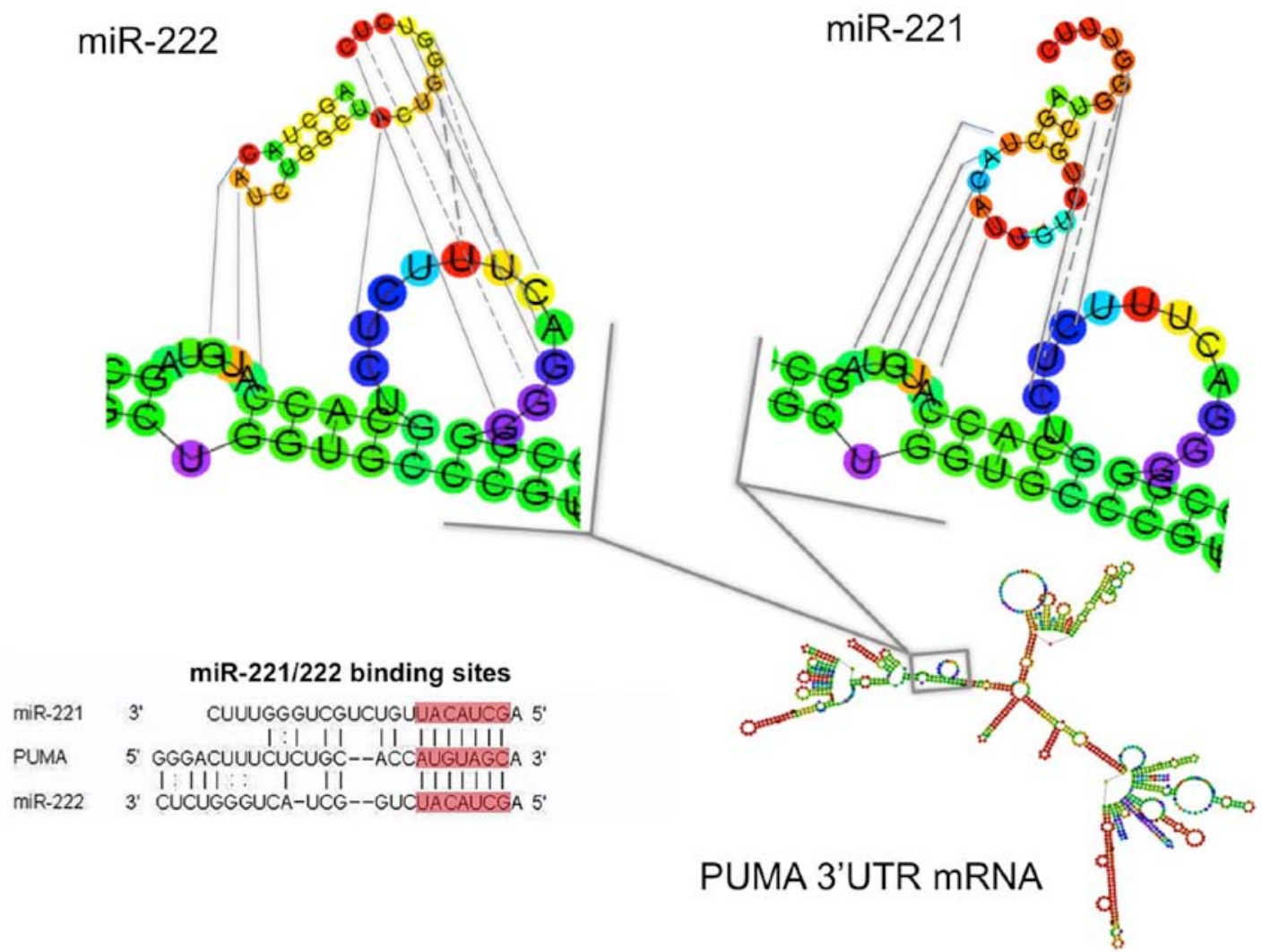

Figure 2. Structures of miR-221, miR-222 and the 3'-UTR sequence of human PUMA mRNA, showing the interactions between miR-221/miR-222 miRNAs and the PUMA 3'-UTR mRNA. In the lower panel, the base-pairing between miR-221, miR-222 and the miR-221/222 PUMA binding site is shown.
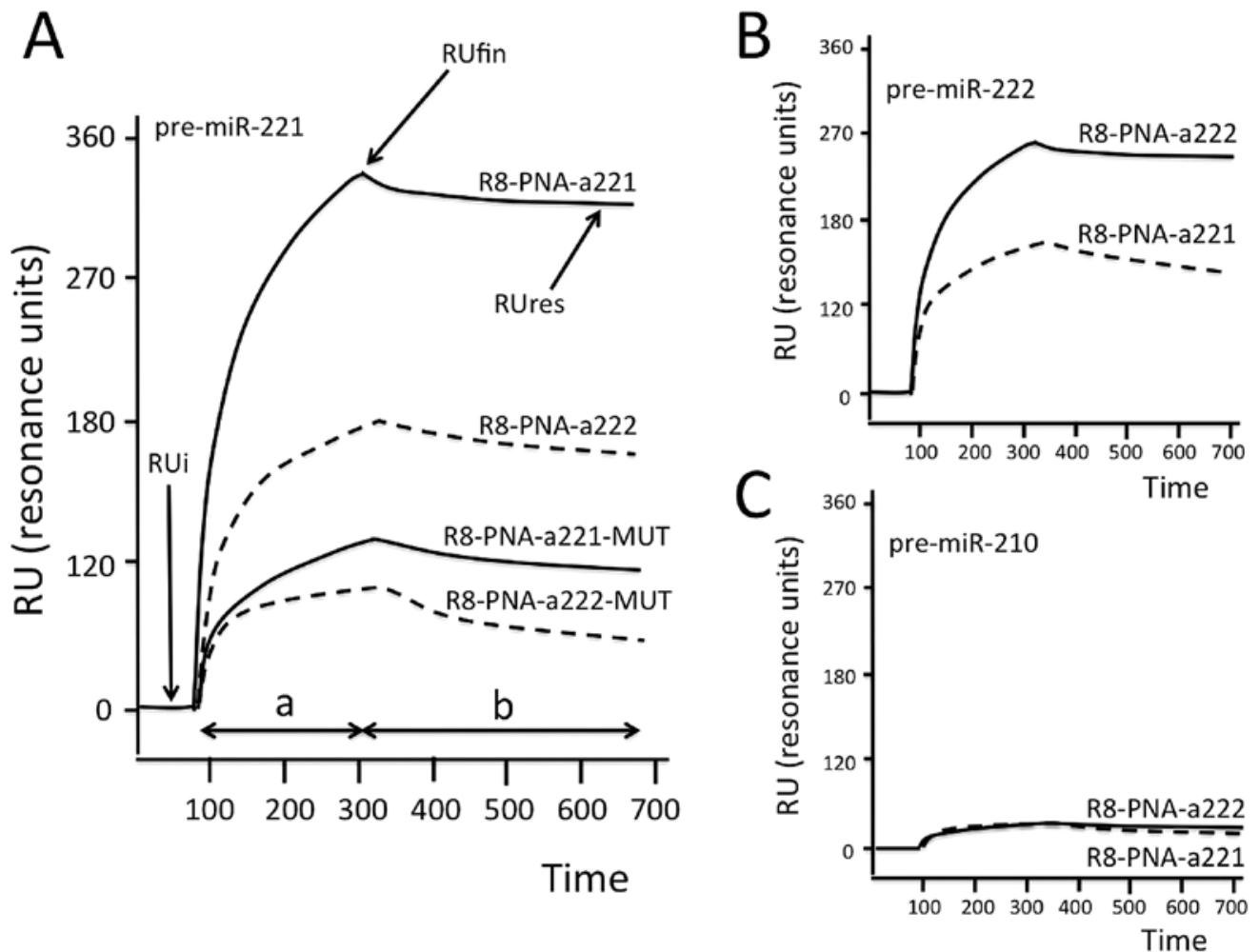

Figure 3. Biospecific interaction analysis. (A) Hybridization between injected pre-miR-221 and sensor-chips to which R8-PNA-a221, R8-PNA-a222, R8-PNAa221-MUT and R8-PNA-a222-MUT were, as indicated, immobilized. The SPR-based Biacore X100 was used. (B and C) BIA showing the hybridization between injected pre-miR-222 (B) and pre-miR-210 (C) and sensor-chips to which R8-PNA-a221 or R8-PNA-a222 were, as indicated, immobilized. RUi, initial RU, i.e., resonance unit (RU) values before the injection; RUfin, final RU after injection; RUres, residual RU after sensor-chip washing with HBS buffer; $a$, analyte injection step; $b$, washing step. Time, seconds. 
Table I. Sequences of PNA used in the present study.

\begin{tabular}{lc}
\hline PNA & \multicolumn{1}{c}{ Sequence } \\
\hline R8-PNA-a221 & H-R8-AAA CCC AGC AGA CAA TGT-Gly-NH ${ }_{2}$ \\
R8-PNA-a221-MUT & H-R8-AAT CCC ACC AGA GAA AGT-Gly-NH ${ }_{2}$ \\
R8-PNA-a222 & H-R8-CAG TAG CCA GAT GTA GCT-Gly-NH ${ }_{2}$ \\
R8-PNA-a222-MUT & H-R8-CAC TAG CGA GAA GTT GCT-Gly-NH ${ }_{2}$ \\
\hline
\end{tabular}

Boldface letters indicate mutated bases.

Table II. Biacore analysis of the hybridization between injected pre-miRNAs and PNAs immobilized on the sensor-chips.

\begin{tabular}{|c|c|c|c|c|}
\hline \multirow[b]{3}{*}{ Immobilized PNAs } & \multicolumn{4}{|c|}{ Injected pre-miRNAs } \\
\hline & \multicolumn{2}{|c|}{ pre-miR-221 } & \multicolumn{2}{|c|}{ pre-miR-222 } \\
\hline & RUfin & RUres & RUfin & RUres \\
\hline R8-PNA-a221 & $301.2 \pm 35.1$ & $300.1 \pm 21.2$ & $163.3 \pm 18.4$ & $144.2 \pm 12.1$ \\
\hline R8-PNA-a221-MUT & $87.4 \pm 7.1$ & $57.2 \pm 6.5$ & $129.8 \pm 9.7$ & $100.1 \pm 9.1$ \\
\hline R8-PNA-a222 & $160.3 \pm 12.5$ & $141.2 \pm 16.1$ & $225.1 \pm 19.2$ & $220.4 \pm 21.2$ \\
\hline R8-PNA-a222-MUT & $54.2 \pm 4.1$ & $42.3 \pm 3.1$ & $140.7 \pm 11.5$ & $126.1 \pm 11.2$ \\
\hline
\end{tabular}

Results represent the average \pm SD of three independent experiments.

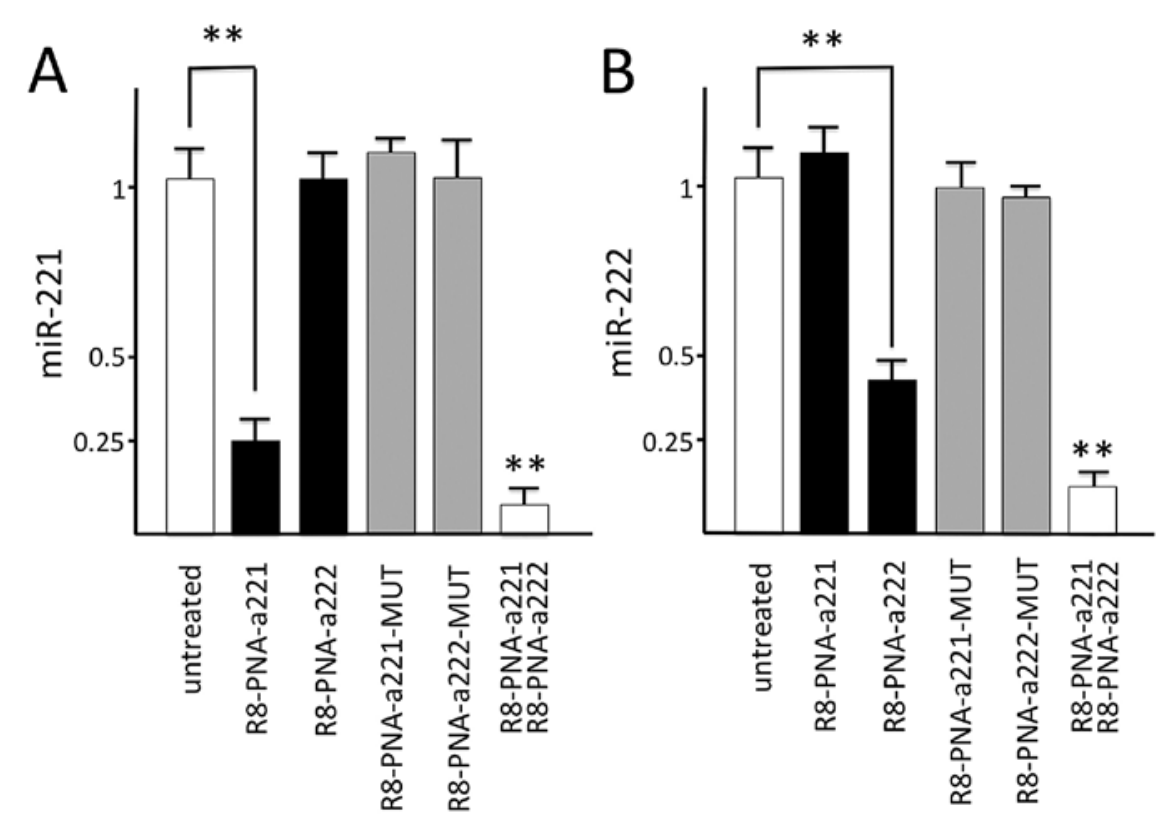

Figure 4. Effects of treatment of U251 cells with $2 \mu \mathrm{M}$ R8-PNA-a221, R8-PNA-a222, R8-PNA-a221-MUT, R8-PNA-a222-MUT and the combination of R8-PNA-a221 and R8-PNA-a222 $(2 \mu \mathrm{M})$. After $48 \mathrm{~h}$ the RNA from untreated and PNA-treated cells was isolated and RT-qPCR performed using AB kits amplifying miR-221 (A) and miR-222 (B) sequences. Data are normalized to the miR-221 (A) and miR-222 (B) levels in untreated cells. Results represent the average \pm SD in three independent experiments.

R8-PNA-a221 and R8-PNA-a222: inhibitory effects on miR-221 and miR-222 in glioma cell lines. When the glioma cell line $\mathrm{U} 251$ was cultured in the presence of the four PNAs, very different effects were obtained, as shown in Fig. 4A. The results demon- strate that the miR-221 signal was strongly reduced only when RNA was isolated from U251 glioma cells cultured for $48 \mathrm{~h}$ in the presence of R8-PNA-a221, while no major effects were observed with R8-PNA-a222. Furthermore, no inhibitory effects were 
A
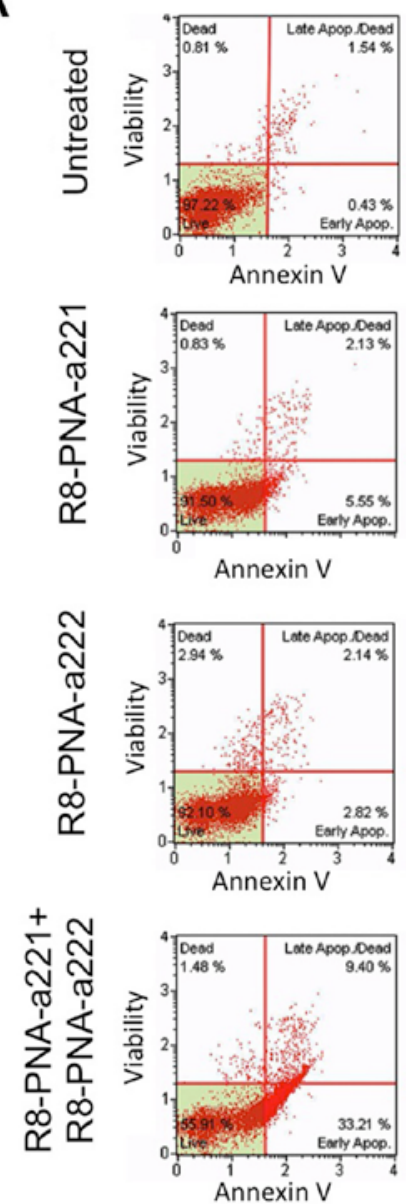

B

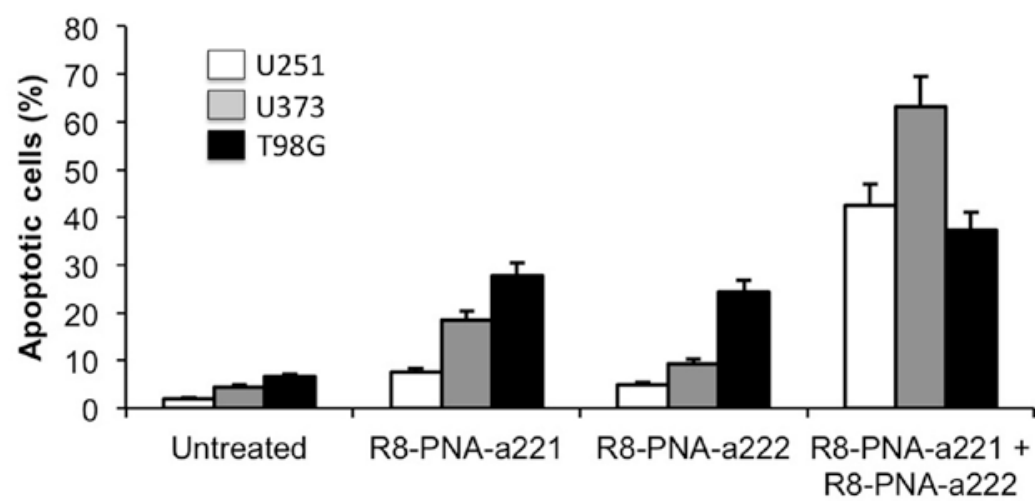

T98G
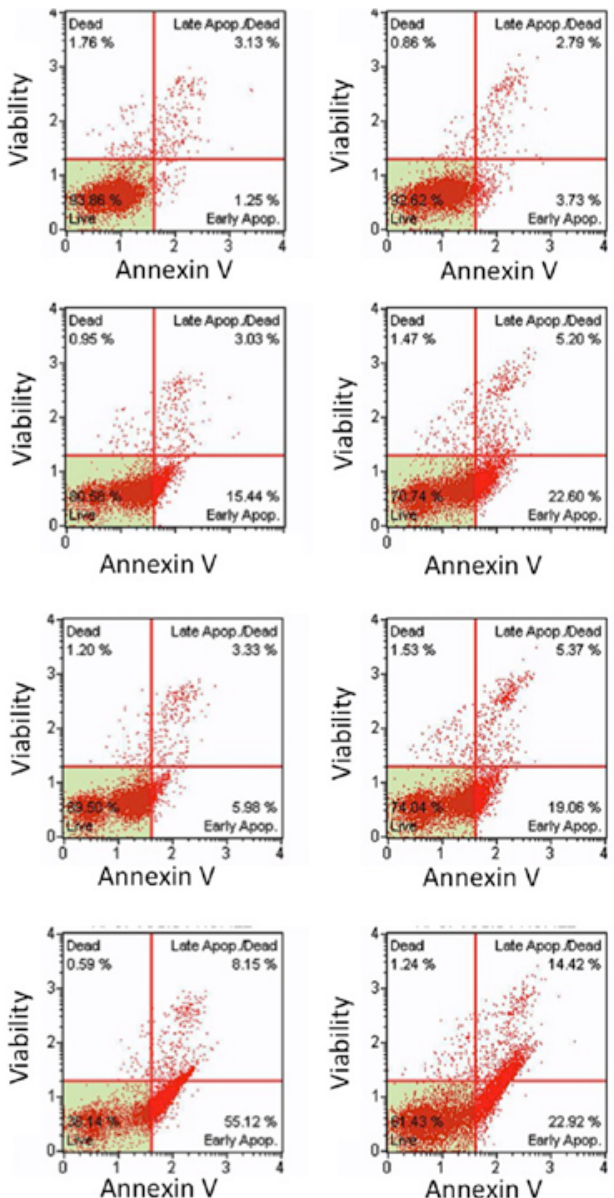

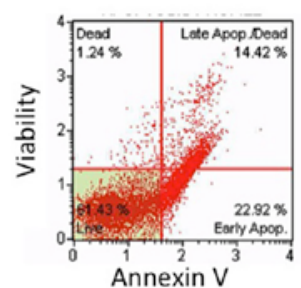

R8-PNA-a222

Figure 5. (A) Increase of apoptotic cells in U251, U373 and T98G glioma cell lines treated for $48 \mathrm{~h}$ with $4 \mu \mathrm{M}$ R8-PNA-a221, with $4 \mu \mathrm{M}$ R8-PNA-a222 and with $2 \mu \mathrm{M}$ R8-PNA-a221 plus with $2 \mu \mathrm{M}$ R8-PNA-a222, as indicated. (B) Summary of the results obtained. The effects of the treatments described in (A) are reported for U251 (white boxes), U373 (grey boxes) and T98G (black boxes). Data represent the sum of the proportion of early and late apoptotic cells (average \pm SD in three different experiments). The apoptosis was determined using the Annexin V and Dead Cell kit with Muse (Millipore Corp.).

observed using R8-PNA-a221-MUT and R8-PNA-a222-MUT. In agreement Fig. 4B shows that miR-222 specific hybridization signal is reduced in samples isolated from U251 cells treated in the presence of R8-PNA-a222, either used alone or in combination with R8-PNA-a221, while no variation is observed after treatment with R8-PNA-a221, R8-PNA-a221-MUT and R8-PNA-a222-MUT. Altogether these experiments support the concept that the effects of R8-PNA-a221 on miR-221 and of R8-PNA-a222 on miR-222 are sequence-specific. The highest effect was obtained after co-treatment with R8-PNA-a221 and R8-PNA-a222.

Co-treatment of U251, U373 and T98G glioma cell lines with R8-PNA-a221 and R8-PNA-a222: effects on apoptosis. When the glioma cell lines U251, U373 and T98G were cultured in the presence of singularly administered R8-PNA-a221 or R8-PNA-a222 a significant and dose-dependent increase of early and late apoptotic cells was observed. Fig. 5A shows 

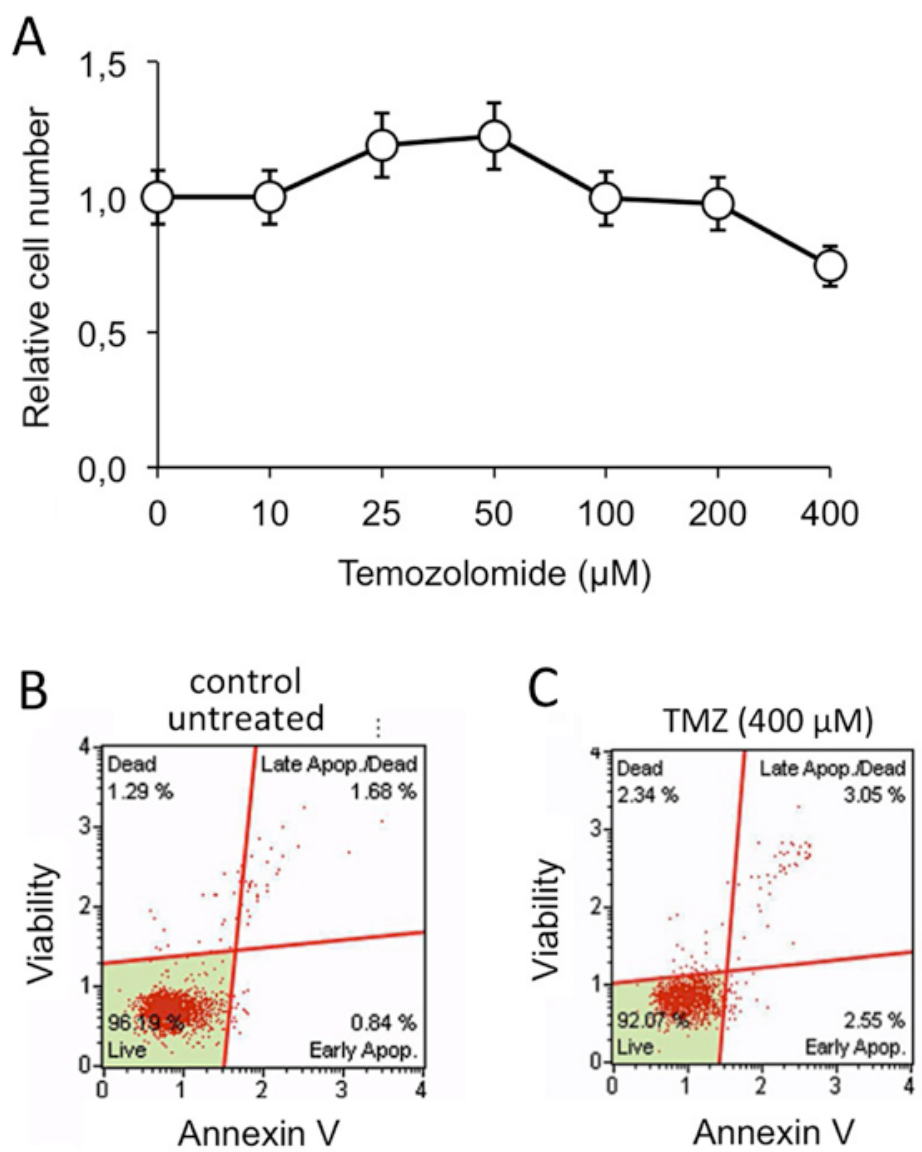

Figure 6. (A) Effects of increasing concentrations (10-400 $\mu \mathrm{M})$ of TMZ on T98G cell growth. Cells were cultured for $72 \mathrm{~h}$ in the different experimental conditions described and then the number of cells/ml counted. (B and C). Lack of pro-apoptotic effects of TMZ. T98G cells were cultured for $48 \mathrm{~h}$ without (B) or with (C) $400 \mu \mathrm{M} \mathrm{TMZ}$ and then the induction of apoptosis determined.

representative results obtained after treatment of $\mathrm{U} 251, \mathrm{U} 373$ and T98G glioma cell lines with $4 \mu \mathrm{M}$ R8-PNA-a221, $4 \mu \mathrm{M}$ R8-PNA-a222, or combined treatment with $2 \mu \mathrm{M}$ R8-PNA-a221 plus $2 \mu \mathrm{M}$ R8-PNA-a222, confirming the pro-apoptotic effects of R8-PNA-a221 [as published by our group (36)] and demonstrating the pro-apoptotic effects of R8-PNA-a222 and the remarkable increased effect obtained with the co-administration of the two PNAs. This effect is superior, especially in the percentage of late apoptotic cells, to that obtained using singularly administered $4 \mu \mathrm{M}$ R8-PNA-a221 and R8-PNA-a222. The full set of data obtained in three independent experiments are comparatively presented in Fig. 5B.

Co-treatment of T98G glioma cells with R8-PNA-a221 and R8-PNA-a222 in the presence of temozolomide (TMZ): effects on apoptosis. As shown in Fig. 6, the T98G cell line is resistant to temozolomide (TMZ) (Fig. 6A) and no increase of apoptosis was observed when these cells were treated with TMZ (Fig. 6B and C). We have previously reported that when TMZ-treatment was conducted in the presence of R8-PNA-a221 a pro-apototic effect was observed much higher than the effect of singularly administered R8-PNA-a221, supporting a possible synergistic effect of TMZ and R8-PNA-a221 on apoptosis of T98G cells (36). Therefore, in our study T98G cells were treated with 2 and $4 \mu \mathrm{M}$ R8-PNA-a221 or R8-PNA-a222 in the presence of $400 \mu \mathrm{M}$ TMZ. The data were compared with the results obtained after $2 \mu \mathrm{M}$ R8-PNA-a221 plus $2 \mu \mathrm{M}$ R8-PNA-a222 treatment in the presence of TMZ. The remarkable results obtained show that co-administration of R8-PNA-a221 and R8-PNA-a222 induced apoptosis of TMZ-treated T98G cells at a level higher than that obtained following singular administration of R8-PNA-a221 or R8-PNA-a222. Representative data are shown in Fig. 7A, the full set of data obtained in three independent experiments are comparatively presented in Fig. 7B.

\section{Discussion}

Gliomas express miR-221 at high levels, promoting malignant progression through activation of the Akt pathway and inhibition of $\mathrm{p} 27^{\mathrm{Kip} 1}$ (44-46); in addition, miR-221 mediated downregulation of other genes such as PUMA (35), ICAM-1 (47), TIMP-3 (48) and PTEN (49) might be associated with glioma onset and progression. Therefore, miR-221 appears to be a specific target for treatments against gliomas (29-35). We have previously reported that a PNA targeting the miR-221 can be internalized by glioma cells when it is linked to an octaarginine tail (R8), leading to inhibition of miR-221 (36). No inhibitory effects were observed on miR-222; moreover, the mutant R8-PNAa221-MUT was inactive in inhibiting miR-221, using RT-qPCR as the validation assay. This effect of anti-miR-221 PNA was associated with the activation of the apoptotic pathway. 

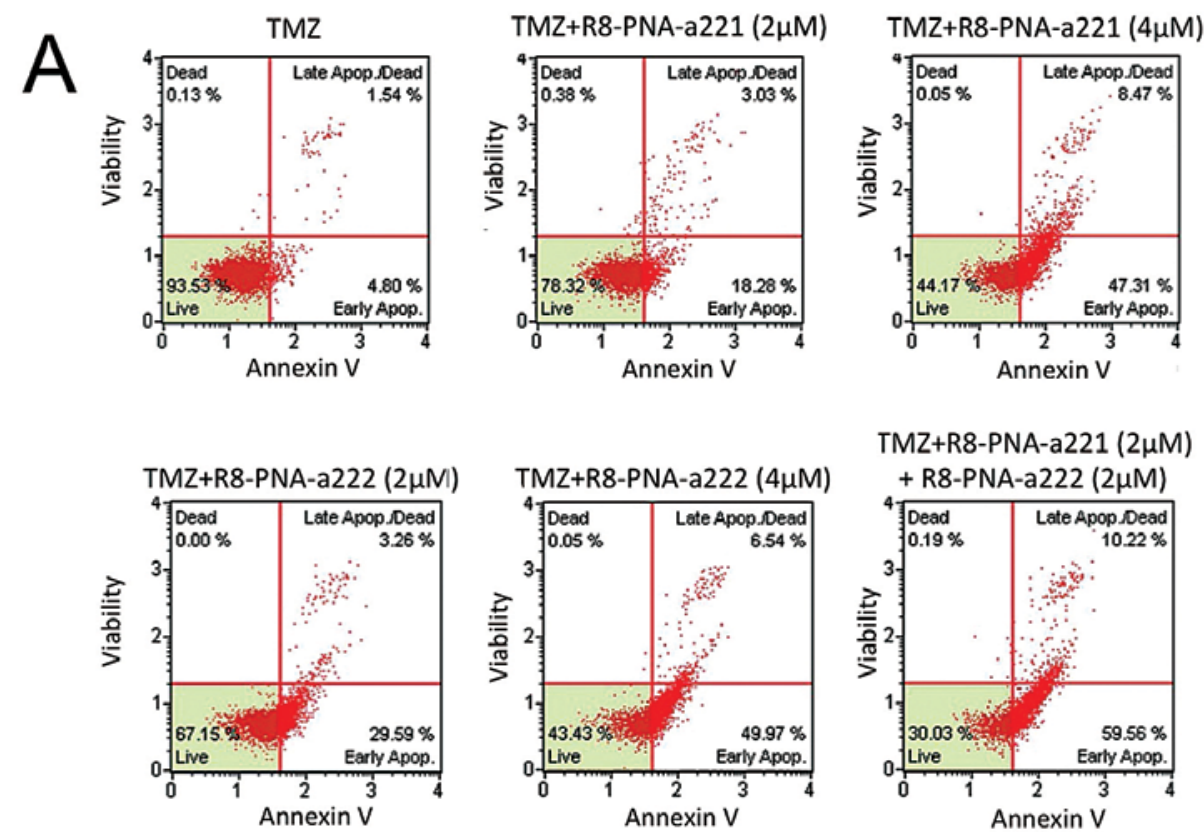

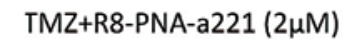

+ R8-PNA-a222 $(2 \mu \mathrm{M})$
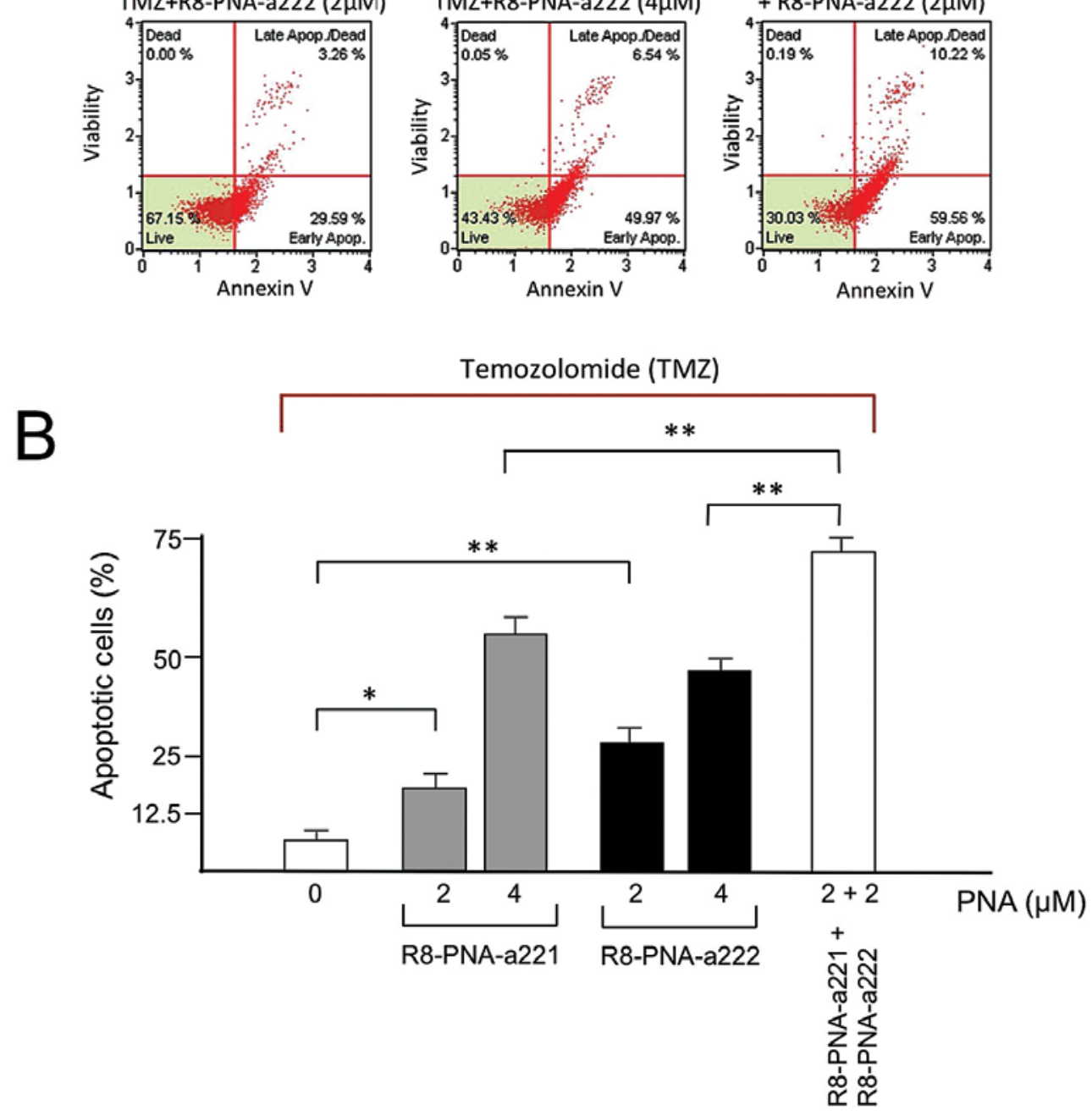

Figure 7. Effects of combined administration of R8-PNA-a221 and R8-PNA-a222 on temozolomide-treated T98G cells. (A) Representative examples of the apoptotic profile obtained in cells treated for $48 \mathrm{~h}$ as indicated and analyzed for Annexin V release. (B) Effects on T98G cells of 2 and $4 \mu \mathrm{M}$ anti-miR PNAs treatments in the presence of temozolomide (TMZ). T98G cells were treated with $400 \mu \mathrm{M} \mathrm{TMZ}$ in the absence (left white box) or in the presence of the indicated concentrations of R8-PNA-a221 (grey columns) and R8-PNA-a222 (black columns). In the white column of the right side of the panel the result obtained after combined treatment with R8-PNA-a221 and R8-PNA-a222 is shown. Results represent the average \pm SD in three independent experiments.

However, the same site recognized by miR-221 in the 3'-UTR of target mRNAs can be also identified by miR-222 (see the example of molecular interactions we have reported in Fig. 2 using PUMA 3'-UTR mRNA as model system). Results very similar to those reported in Fig. 2 can be obtained using 3'-UTR sequences of other miR-221 target mRNAs, such as p27 $7^{\text {Kip1 }}$, PTEN and TIMP-3 (data not shown). Therefore, targeting of miR-221 with anti-miRs, including PNA-based anti-miR, might not be sufficient to obtain full suppression of
miR-221 biological activity due to the presence of miR-222 in target cells. Since miR-221 and miR-222 belong to a same transcriptional unit and are, as expected, co-expressed in glioma cell lines, including those used in the present report, we were interested to determine whether co-administration of anti-miR peptide nucleic acids recognizing miR-221 and miR-222 leads to more efficient inhibitory activity on miR-221/222 dependent functions, promoting pro-apoptotic effects on brain tumor cells. 
The major results of the present investigation are the following: a) R8-conjugated PNAs show biological activity against miR-221 (R8-PNA-a221) and miR-222 (R8-PNA-a222); b) SPR-BIA analysis shows that the recognition of R8-PNA-a221 is selective for miR-221 sequences and that of R8-PNA-a222 is selective for miR-222, with only partial cross-binding; c) no interactions of miR-221 and miR-222 occurs with mutated R8-PNA-a221-MUT and R8-PNA-a222-MUT, respectively; d) when R8-PNA-a221 and R8-PNA-a222 are singularly administered to glioma cells, specific inhibition of hybridization to miR-221 and miR-222, respectively, are obtained following RT-qPCR analysis; e) both R8-PNA-a221 and R8-PNA-a222 induce apoptosis of U251, U373 and T98G glioma cell lines; f) the treatment of T98G cells with R8-PNA-a221 and R8-PNA-a222 reversed the resistance of the cells to apoptosis induced by temozolomide (TMZ); g) when the R8-PNA-a221 and R8-PNA-a222 are co-administered the pro-apoptotic effects on U251, U373 and T98G cells and the reversion of TMZ resistance in T98G cells are much more evident.

In conclusion, our results support the concept that antimiR strategy led to therapeutic relevant inhibition of miRNA dependent effects $(18,20,21,25-28)$ and that PNA-based anti$\mathrm{miR}$ molecules are very promising reagents to regulate tumor cell growth; further research on PNA analogues to increase efficiency of delivery, stability and control of intracellular distribution for specific targets, i.e., mature miRNA, pre-miRNA or pri-miRNA, are further steps for the selection of best candidate drugs. Finally, our study strongly indicates that the combined treatment of tumor cells with PNAs targeting both miR-221 and miR-222, or, more generally, multiple miR targets, might lead to significant improvement in the efficacy of the treatment. This last conclusion supports also the concept of designing multifunctional PNA-containing systems or nanocarriers (50), enabling to perform targeting of different miRNA sequences.

\section{Acknowledgements}

This study was supported by CIB, by COFIN-2009 and by AIRC (IG 13575: peptide nucleic acids targeting oncomiR and tumor-suppressor miRNAs: cancer diagnosis and therapy). G.C. was funded by Verona Brain Research Foundation.

\section{References}

1. Nielsen PE, Egholm M, Berg RH and Buchardt O: Sequenceselective recognition of DNA by strand displacement with a thymine-substituted polyamide. Science 254: 1497-1500, 1991.

2. Nielsen PE: Targeting double stranded DNA with peptide nucleic acid (PNA). Curr Med Chem 8: 545-550, 2001.

3. Borgatti M, Lampronti I, Romanelli A, Pedone C, Saviano M, Bianchi N, Mischiati C and Gambari R: Transcription factor decoy molecules based on a peptide nucleic acid (PNA)-DNA chimera mimicking Sp1 binding sites. J Biol Chem 278: 7500-7509, 2003.

4. Gambari R: Peptide-nucleic acids (PNAs): A tool for the development of gene expression modifiers. Curr Pharm Des 7: 1839-1862, 2001.

5. Gambari R: Biological activity and delivery of peptide nucleic acids (PNA)-DNA chimeras for transcription factor decoy (TFD) pharmacotherapy. Curr Med Chem 11: 1253-1263, 2004.

6. Nielsen PE: Peptide nucleic acids (PNA) in chemical biology and drug discovery. Chem Biodivers 7: 786-804, 2010.

7. Hatamoto M, Ohashi A and Imachi H: Peptide nucleic acids (PNAs) antisense effect to bacterial growth and their application potentiality in biotechnology. Appl Microbiol Biotechnol 86: 397-402, 2010.
8. Gambari R, Borgatti M, Bezzerri V, Nicolis E, Lampronti I, Dechecchi MC, Mancini I, Tamanini A and Cabrini G: Decoy oligodeoxyribonucleotides and peptide nucleic acids-DNA chimeras targeting nuclear factor kappa-B: Inhibition of IL-8 gene expression in cystic fibrosis cells infected with Pseudomonas aeruginosa. Biochem Pharmacol 80: 1887-1894, 2010.

9. Pandey VN, Upadhyay A and Chaubey B: Prospects for antisense peptide nucleic acid (PNA) therapies for HIV. Expert Opin Biol Ther 9: 975-989, 2009.

10. Nielsen PE: Gene targeting and expression modulation by peptide nucleic acids (PNA). Curr Pharm Des 16: 3118-3123, 2010.

11. Manicardi A, Fabbri E, Tedeschi T, Sforza S, Bianchi N, Brognara E, Gambari R, Marchelli R and Corradini R: Cellular uptakes, biostabilities and anti-miR-210 activities of chiral arginine-PNAs in leukaemic K562 cells. ChemBioChem 13: 1327-1337, 2012.

12. Fabbri E, Manicardi A, Tedeschi T, Sforza S, Bianchi N, Brognara E, Finotti A, Breveglieri G, Borgatti M, Corradini R, et al: Modulation of the biological activity of microRNA-210 with peptide nucleic acids (PNAs). ChemMedChem 6: 2192-2202, 2011.

13. Gambari R, Fabbri E, Borgatti M, Lampronti I, Finotti A, Brognara E, Bianchi N, Manicardi A, Marchelli R and Corradini R: Targeting microRNAs involved in human diseases: A novel approach for modification of gene expression and drug development. Biochem Pharmacol 82: 1416-1429, 2011.

14. Fabani MM and Gait MJ: miR-122 targeting with LNA/2 $\square-\mathrm{O}-$ methyl oligonucleotide mixmers, peptide nucleic acids (PNA), and PNA-peptide conjugates. RNA 14: 336-346, 2008.

15. Fabani MM, Abreu-Goodger C, Williams D, Lyons PA, Torres AG, Smith KG, Enright AJ, Gait MJ and Vigorito E: Efficient inhibition of miR-155 function in vivo by peptide nucleic acids. Nucleic Acids Res 38: 4466-4475, 2010.

16. Brown PN and Yin H: PNA-based microRNA inhibitors elicit anti-inflammatory effects in microglia cells. Chem Commun (Camb) 49: 4415-4417, 2013.

17. Brognara E, Fabbri E, Aimi F, Manicardi A, Bianchi N, Finotti A, Breveglieri G, Borgatti M, Corradini R, Marchelli R, et al: Peptide nucleic acids targeting miR-221 modulate $\mathrm{p} 27^{\mathrm{Kip}}$ expression in breast cancer MDA-MB-231 cells. Int J Oncol 41: 2119-2127, 2012.

18. Cheng CJ, Bahal R, Babar IA, Pincus Z, Barrera F, Liu C, Svoronos A, Braddock DT, Glazer PM, Engelman DM, et al: MicroRNA silencing for cancer therapy targeted to the tumour microenvironment. Nature 518: 107-110, 2015.

19. He L and Hannon GJ: MicroRNAs: Small RNAs with a big role in gene regulation. Nat Rev Genet 5: 522-531, 2004.

20. Piva R, Spandidos DA and Gambari R: From microRNA functions to microRNA therapeutics: Novel targets and novel drugs in breast cancer research and treatment (Review). Int J Oncol 43: 985-994, 2013.

21. Taylor MA and Schiemann WP: Therapeutic opportunities for targeting microRNAs in cancer. Mol Cell Ther 2: 1-13, 2014.

22. Song MS and Rossi JJ: The anti-miR21 antagomir, a therapeutic tool for colorectal cancer, has a potential synergistic effect by perturbing an angiogenesis-associated miR30. Front Genet 4: 301, 2014

23. Nana-Sinkam SP and Croce CM: Clinical applications for microRNAs in cancer. Clin Pharmacol Ther 93: 98-104, 2013.

24. Hermansen SK and Kristensen BW: MicroRNA biomarkers in glioblastoma. J Neurooncol 114: 13-23, 2013.

25. Shu M, Zheng X, Wu S, Lu H, Leng T, Zhu W, Zhou Y, Ou Y, Lin X, Lin Y, et al: Targeting oncogenic miR-335 inhibits growth and invasion of malignant astrocytoma cells. Mol Cancer 10: 59, 2011.

26. Chan XH, Nama S, Gopal F, Rizk P, Ramasamy S, Sundaram G, Ow GS, Ivshina AV, Tanavde V, Haybaeck J, et al: Targeting glioma stem cells by functional inhibition of a prosurvival oncomiR-138 in malignant gliomas. Cell Reports 2: 591-602, 2012.

27. Wagenaar TR, Zabludoff S, Ahn SM, Allerson C, Arlt H, Baffa R, Cao H, Davis S, Garcia-Echeverria C, Gaur R, et al: Anti-miR-21 suppresses hepatocellular carcinoma growth via broad transcriptional network de-regulation. Mol Cancer Res 13: 1009-1021, 2015

28. Ma L, Reinhardt F, Pan E, Soutschek J, Bhat B, Marcusson EG, Teruya-Feldstein J, Bell GW and Weinberg RA: Therapeutic silencing of miR-10b inhibits metastasis in a mouse mammary tumor model. Nat Biotechnol 28: 341-347, 2010. 
29. Shah MY and Calin GA: MicroRNAs miR-221 and miR-222: A new level of regulation in aggressive breast cancer. Genome Med 3: 56,2011

30. Lambertini E, Lolli A, Vezzali F, Penolazzi L, Gambari R and Piva R: Correlation between Slug transcription factor and miR-221 in MDA-MB-231 breast cancer cells. BMC Cancer 12: 445, 2012.

31. Galardi S, Mercatelli N, Giorda E, Massalini S, Frajese GV, Ciafrè SA and Farace MG: miR-221 and miR-222 expression affects the proliferation potential of human prostate carcinoma cell lines by targeting p27 ${ }^{\mathrm{Kip} 1}$. J Biol Chem 282: 23716-23724, 2007.

32. Yang J,Zhang JY, Chen J, Xu Y, Song NH and Yin CJ: Prognostic role of microRNA-221 in various human malignant neoplasms: A meta-analysis of 20 related studies. PLoS One 9: e87606, 2014

33. Li W, Guo F, Wang P, Hong S and Zhang C: miR-221/222 confers radioresistance in glioblastoma cells through activating Akt independent of PTEN status. Curr Mol Med 14: 185-195, 2014.

34. Chen L, Zhang J, Han L, Zhang A, Zhang C, Zheng Y, Jiang T, $\mathrm{Pu}$ P, Jiang C and Kang C: Downregulation of miR-221/222 sensitizes glioma cells to temozolomide by regulating apoptosis independently of p53 status. Oncol Rep 27: 854-860, 2012.

35. Zhang CZ, Zhang JX, Zhang AL, Shi ZD, Han L, Jia ZF, Yang WD, Wang GX, Jiang T, You YP, et al: MiR-221 and miR-222 target PUMA to induce cell survival in glioblastoma. Mol Cancer 9: 229, 2010.

36. Brognara E, Fabbri E, Bazzoli E, Montagner G, Ghimenton C, Eccher A, Cantù C, Manicardi A, Bianchi N, Finotti A, et al: Uptake by human glioma cell lines and biological effects of a peptide-nucleic acids targeting miR-221. J Neurooncol 118: 19-28, 2014.

37. Jensen KK, Orum H, Nielsen PE and Nordén B: Kinetics for hybridization of peptide nucleic acids (PNA) with DNA and RNA studied with the BIAcore technique. Biochemistry 36 5072-5077, 1997.

38. Corradini R, Feriotto G, Sforza S, Marchelli R and Gambari R: Enhanced recognition of cystic fibrosis W1282X DNA point mutation by chiral peptide nucleic acid probes by a surface plasmon resonance biosensor. J Mol Recognit 17: 76-84, 2004

39. Cao X, Gu Y, Jiang L, Wang Y, Liu F, Xu Y, Deng J, Nan Y, Zhang L, Ye J, et al: A new approach to screening cancer stem cells from the U251 human glioma cell line based on cell growth state. Oncol Rep 29: 1013-1018, 2013.
40. Abdullah Thani NA, Sallis B, Nuttall R, Schubert FR, Ahsan M, Davies D, Purewal S, Cooper A and Rooprai HK: Induction of apoptosis and reduction of MMP gene expression in the U373 cell line by polyphenolics in Aronia melanocarpa and by curcumin. Oncol Rep 28: 1435-1442, 2012.

41. Pen A, Durocher Y, Slinn J, Rukhlova M, Charlebois C, Stanimirovic DB and Moreno MJ: Insulin-like growth factor binding protein 7 exhibits tumor suppressive and vessel stabilization properties in U87MG and T98G glioblastoma cell lines. Cancer Biol Ther 12: 634-646, 2011.

42. Rothbard JB, Kreider E, VanDeusen CL, Wright L, Wylie BL and Wender PA: Arginine-rich molecular transporters for drug delivery: Role of backbone spacing in cellular uptake. J Med Chem 45: 3612-3618, 2002.

43. Abes R, Arzumanov A, Moulton H, Abes S, Ivanova G, Gait MJ Iversen $\mathrm{P}$ and Lebleu B: Arginine-rich cell penetrating peptides: Design, structure-activity, and applications to alter pre-mRNA splicing by steric-block oligonucleotides. J Pept Sci 14: 455-460, 2008.

44. Bhatia B: On the move: $\mathrm{p} 27^{\mathrm{Kip} 1}$ drives cell motility in glioma cells. Cell Cycle 9: 1231-1240, 2010.

45. Lu X, Zhao P, Zhang C, Fu Z, Chen Y, Lu A, Liu N, You Y, Pu P and Kang C: Analysis of miR-221 and p27 expression in human gliomas. Mol Med Rep 2: 651-656, 2009.

46. Gillies JK and Lorimer IA: Regulation of $\mathrm{p} 27^{\mathrm{Kip} 1}$ by miRNA 221/222 in glioblastoma. Cell Cycle 6: 2005-2009, 2007.

47. Ueda R, Kohanbash G, Sasaki K, Fujita M,Zhu X, KastenhuberER, McDonald HA, Potter DM, Hamilton RL, Lotze MT, et al: Dicerregulated microRNAs 222 and 339 promote resistance of cancer cells to cytotoxic T-lymphocytes by down-regulation of ICAM-1. Proc Natl Acad Sci USA 106: 10746-10751, 2009.

48. Zerrouqi A, Pyrzynska B, Febbraio M, Brat DJ and Van Meir EG: P14ARF inhibits human glioblastoma-induced angiogenesis by upregulating the expression of TIMP3. J Clin Invest 122: 1283-1295, 2012 .

49. Wang Y, Wang X, Zhang J, Sun G, Luo H, Kang C, Pu P, Jiang T, Liu N and You Y: MicroRNAs involved in the EGFR/PTEN/ AKT pathway in gliomas. J Neurooncol 106: 217-224, 2012.

50. Bertucci A, Lülf H, Septiadi D, Manicardi A, Corradini R and De Cola L: Intracellular delivery of peptide nucleic acid and organic molecules using zeolite-L nanocrystals. Adv Healthcare Mater 3: 1812-1817, 2014. 